\title{
A taxonomic analysis of fungi collected and described from Egypt up to 1931
}

\section{Soliman EA ${ }^{1}$, Abdel-Azeem AM ${ }^{1,2}$, Salem FM ${ }^{1,2,3}$, Nafady NA ${ }^{1,4}$, Mehesien MT $^{1,5}$, Ahmed AIS ${ }^{1,3}$, Ibrahem ME ${ }^{6}$, Abdel-Azeem MA ${ }^{1,7}$ and Hassan SS ${ }^{1,2}$}

${ }^{1}$ Arab Society for Fungal Conservation, Botany Department, Faculty of Science, University of Suez Canal, Ismailia 41522, Egypt.

${ }^{2}$ Botany Department, Faculty of Science, University of Suez Canal, Ismailia 41522, Egypt.

${ }^{3}$ Plant Pathology Unit, Plant Protection Department, Desert Research Center, El-Matarya, Cairo, Egypt

${ }^{4}$ Botany and Microbiology Department, Faculty of Science, Assuit University, Assiut 71516, Egypt.

${ }^{5}$ Botany Department, Faculty of Science, University of Damietta, New Damietta, Egypt.

${ }^{6}$ Botany Department, Faculty of Science, University of Port Said, Port Said, Egypt.

${ }^{7}$ Faculty of Pharmacy and Pharmaceutical Industry, University of Sinai, Al-Arish, El Masaid, .North Sinai, Egypt.

Soliman EA, Abdel-Azeem AM, Salem FM, Nafady NA, Mehesien MT, Ahmed AIS, Ibrahem ME, Abdel-Azeem MA, Hassan SS 2016 - A taxonomic analysis of fungi collected and described from Egypt up to 1931. Studies in Fungi 1(1), 11-33, Doi 10.5943/sif/1/1/2

\begin{abstract}
A revisit of 13 publications published since 1813 to 1931 is presented and the 340 taxa reported are taxonomically re-evaluated. The Egyptian taxa treated belong to two kingdoms and three major groups: zygomycetes (1 spp.), ascomycetes (124 spp.), basidiomycetes (197 spp.) and chromistian fungal analogues (18 species). Taxa of true fungi are distributed among three phyla, ten classes, 38 orders, and 67 families, while Chromistean fungal analogues distributed in only one phylum, two orders and three families. About $35 \%$ of the original names of taxa have undergone changes, although for a few new designations are still debatable. The binomials of the remaining taxa are unchanged. Thus despite it being over 200 years since publication of Delile on Egyptian fungi and the present analysis, the taxonomic status of many Egyptian taxa, awaits re-assessment in modern terms.
\end{abstract}

Key words - Arid - ascomycetes - basidiomycetes - biodiversity - fungi- nomenclature taxonomy

\section{Introduction}

Saprobic and phytopathogenic fungi on wild and domesticated plants of economic importance have been attracted the attention of many researchers in the late $19^{\text {th }} /$ early $20^{\text {th }}$ century in Egypt e.g. Delile (1813), Thüemen (1878, 1879, 1880), Ascherson (1879), Roumeguère (1881, 1887), Sickenberger (1901), Fletcher (1902), Reichert (1921), Fahmy (1923), Shearer (1924), Briton-Jones (1922, 1923, 1925), Bishara (1928) and Melchers (1931).

Works of Reichert and Melchers were considered the starting date of the documentation of Egyptian phytopathogenic fungi. Israel Reichert (1891-1975) obtained his doctorate on Die Pilzflora Ägypten in which 237 species were recognized, of which 42 were new to science without any specimens were retained in Egypt. He used material collected before 1914 found in the 
Botanisches Museum in Berlin-Dahlem, which Reichert used when compiling his list of 1921, but it is not known if these specimens survived World War II (Abdel-Azeem 2010). A check list of plant diseases and fungi occurring in Egypt published by Melchers (1931) in Transactions of the Kansas Academy of Science is considered the valuable update of aforementioned studies and species list. Melchers went to Egypt in 1927 as chief mycologist for 18 months by the invitation of the Egyptian Minister of Agriculture. His checklist included 345 species of fungi, especially those causing plant diseases and he met a series of difficulties such as there being no records available on the occurrence, distribution, or dates of any mycological observations conducted previously by any investigator in Egypt and no mycological reference collection (Melchers 1931).

Taxonomy requires continual updates and checklists. Checklists are important tools in taxonomy, systematics and conservation (Söderström et al. 2007, 2008). Records of Egyptian fungi, are scattered through a wide array of journals, books, and dissertations, but preliminary annotated checklists and compilations are not all readily available and this is the main reason that Egypt lacks recent checklists (Abdel-Azeem 2010, Abdel-Azeem \& Salem, 2013). Only very few comprehensive assessments of Egyptian fungi have been published in the $20^{\text {th }}$ and $21^{\text {st }}$ centuries e.g. El-Abyad and Abu-Taleb 1993; El-Abyad 1997; Abdel-Azeem, 2010, Moustafa \& AbdelAzeem 2011, Abdel-Azeem \& Salem 2013).

\section{Materials \& Methods}

The main target of this study is the $19^{\text {th }}$ and early $20^{\text {th }}$ collections of Egyptian fungi initially identified by Delile (1813), Decaisne (1835), Ascherson (1879), Thümen (1878, 1879, 1880), Roumeguère (1881, 1887), Patouillard (1895), Sickenberger (1901), Magnus (1910), Reichert (1921), and Melchers (1931). These fungi and their descriptions are hardly accessible or traceable, however, a systematic review of these taxa is long overdue. Therefore, the classification of these fungi are now updated and resubmitted below, together with their original data to provide a contemporary and comparative taxonomic study. The names of authors of fungal taxa are abbreviated according to Kirk and Ansell (1992). The systematic arrangement of the recorded list follows the $10^{\text {th }}$ edition of Anisworth and Bisby's Dictionary of the Fungi (Kirk et al. 2008). All name corrections, authorities, and taxonomic assignments of recorded species in this study were checked against the Index Fungorum database (www. indexfungorum.org).

\section{Results}

One hundred and forty-seven genera and 340 (119 synonmized) species were recorded during this period. Taxa of true fungi distributed among three phyla, ten classes, 38 orders, and 67 families, while Chromistean fungal analogues are distributed in only one phylum, two orders and three families. Taxa with uncertain position are distributed among classes, orders and families. The records are reviewed and are enumerated below in alphabetical and hierarchical order. Species of each group are given in a taxonomic sequence and accepted names are highlighted in bold. Classification of Dothideomycetes was followed Hyde et al. (2013) and Wijayawardene et al. (2014).

Botryosphaeriaceae, Botryosphaeriales

\section{Ascomycota, Dothideomycetes}

1. Diplodia dracaenae Henn., Hedwigia 48: 112 (1908)

Ref.: Melchers 1931.

2. Diplodia opuntiae Sacc., Michelia 2 (no. 7): 267 (1881)

Ref.: Reichert 1921, Melchers 1931.

3. Diplodia warburgiana Reichert, Bot. Jb. 56: 715 (1921)

Ref.: Reichert 1921, Melchers 1931.

4. Macrophoma engleriana Reichert, Bot. Jb. 56: 712 (1921)

Ref.: Reichert 1921, Melchers 1931.

5. Microdiplodia machlaiana Reichert, Bot. Jb. 56: 714 (1921) 
Ref.: Reichert 1921, Melchers 1931.

6. Sphaeropsis calotropidis Thüm., in Schweinfurth \& Thümen, Grevillea 8 (no. 46): 51 (1880)

Ref.: Thüemen 1879, 1880.

7. Phaeobotryosphaeria visci (Kalchbr.) A.J.L. Phillips \& Crous, in Phillips, Alves, Pennycook, Johnston, Ramaley, Akulov \& Crous, Persoonia 21: 47 (2008)

Synonym: - Sphaeropsis visci (Alb. \& Schwein.) Sacc., Michelia 2 (no. 6): 105 (1880)

Ref.: Melchers 1931.

Phyllostictaceae, Botryosphaeriales,

8. Guignardia egyptiaca (Müll. Arg.) Reichert, Bot. Jb. 56: 669 (1921)

Ref.: Reichert 1921, Melchers 1931.

9. Phyllosticta gossypina Ellis \& G. Martin, J. Mycol. 2(11): 129 (1886)

Ref.: Melchers 1931.

10. Phyllosticta palmarum Rabenh., Fungi europ. exsicc., Edn nova. Cent. 2: no. 2161 (1876)

Ref.: Reichert 1921, Melchers 1931.

\section{Capnodiaceae, Capnodiales}

11. Capnodium citricola McAlpine, Proc. Linn. Soc. of New S. Wales 1869, part IV, p. 491 (1896)

Ref.: Melchers 1931.

Cladosporiaceae, Capnodiales,

12. Cladosporium acaciae Reichert, Bot. Jb. 56: 720 (1921)

Ref.: Reichert 1921, Melchers 1931.

13. Cladosporium herbarum (Pers.) Link, in Willdenow, Mag. Gesell. naturf. Freunde, Berlin 8: 37 (1816).

Ref.: Thüemen 1880, Roumeguère 1881, Sickenberger 1901, Reichert 1921, Melchers 1931.

14. Cladosporium hibisci Reichert, Bot. Jb. 56: 721 (1921)

Ref.: Reichert 1921, Melchers 1931.

15. Cladosporium pyriforme Reichert Bot. Jb. 56: 721 (1921)

Ref.: Reichert 1921, Melchers 1931.

16. Cladosporium typharum Desm., Pl. crypt. exsicc. 1: no. 304 (1834)

Ref.: Sickenberger 1901, Reichert 1921, Melchers 1931.

Capnodiales genera, incertae sedis

17. Torula herbarum (Pers.) Link, Mag. Gesell. naturf. Freunde, Berlin 3(1-2): 19 (1809)

Ref.: Sickenberger 1901, Reichert 1921, Melchers 1931.

18. Torula opuntiae Reichert, Bot. Jb. 56: 718 (1921)

Ref.: Reichert 1921, Melchers 1931.

Mycosphaerellaceae, Capnodiales,

19. Cercospora apii Fresen., Beitr. Mykol. 3: 91 (1863)

Ref.: Melchers 1931.

20. Cercospora beticola Sacc., Nuovo G. bot. ital. 8 (2): 189 (1876)

Ref.: Melchers 1931.

21. Cercospora roesleri (Catt.) Sacc., Michelia 2 (no. 6): 128 (1880)

Ref.: Reichert 1921, Melchers 1931.

22. Cercospora violae Sacc., Nuovo G. bot. ital. 8 (2): 187 (1876),

Ref.: Reichert 1921- Melchers 1931.

23. Cymadothea trifolii (Pers.) F.A. Wolf, Mycologia 27(1): 71 (1935)

Synonym: - Phyllachora trifolii (Pers.) Fuckel, Jb. nassau. Ver. Naturk. 23-24: 218 (1870)

[1869-70]

- Polythrincium trifolii Kunze, in Kunze \& Schmidt, Mykologische Hefte (Leipzig) 1: 14 (1817)

Ref.: Magnus 1910, Reichert 1921, Melchers 1931. 
24. Mycosphaerella berkeleyi W.A. Jenkins, J. Agric. Res., Washington 56: 325 (1938)

Synonym: -Cercospora personata (Berk. \& M.A. Curtis) Ellis, J. Mycol. 1(5): 63 (1885) Ref.: Melchers 1931.

25. Mycosphaerella engleriana Reichert, Bot. Jb. 56: 670 (1921) Ref.: Reichert 1921, Melchers 1931.

26. Mycosphaerella fragariae (Tul. \& C. Tul.) Lindau, in Engler \& Prantl, Nat. Pflanzenfam., Teil. I (Leipzig) 1(1): 424 (1897)

Ref.: Melchers 1931.

27. Mycosphaerella tassiana (De Not.) Johanson, Öfvers. K. Svensk. Vetensk.-Akad. Förhandl. 41(no. 9): 167 (1884)

Synonym: - Cladosporium graminum (Pers.) Link, in Willdenow, Sp. pl., Edn 4 6(1): 42 (1824)

Ref.: Reichert 1921, Melchers 1931.

28. Passalora fulva (Cooke) U. Braun \& Crous, in Crous \& Braun, CBS Diversity Ser. (Utrecht) 1: 453 (2003)

Ref.: Melchers 1931.

29. Pseudocercospora snelliana (Reichert) U. Braun, H.D. Shin, C. Nakash. \& Crous, in Crous, Braun, Hunter, Wingfield, Verkley, Shin, Nakashima \& Groenewald, Stud. Mycol. 75: 106 (2012)

Synonym: -Cercospora snelliana Reichert, Bot. Jb. 56: 724 (1921)

Ref.: Reichert 1921.

30. Pseudocercospora vitis (Lév.) Speg., Anal. Mus. nac. B. Aires, Ser. 3 13: 438 (1911) Ref.: Reichert 1921.

31. Sphaerella carlii Fuckel, Jb. nassau. Ver. Naturk. 23-24: 103 (1870) Ref.: Thüemen 1880.

32. Stigmidium schaereri (A. Massal.) Trevis., Conspect. Verruc.: 17 (1860)

Synonym: - Pharcidia epicymatia (Wallr.) G. Winter, Rabenh. Krypt.-Fl., Edn 2 (Leipzig) 1.2: 342 (1885)

Ref.: Sickenberger 1901, Reichert 1921, Melchers 1931.

33. Stigmina carpophila (Lév.) M.B. Ellis, Mycol. Pap. 72: 56 (1959)

Synonym: -Coryneum beijerinckii Oudem., Hedwigia 22: 115 (1883)

Ref.: Melchers 1931.

Dothideaceae, Dothideales

34. Scirrhia rimosa (Alb. \& Schwein.) Fuckel, Jb. nassau. Ver. Naturk. 23-24: 221 (1870) Ref.: Reichert 1921, Melchers 1931.

Gloniaceae, Mytilinidiales

35. Glonium guttulatum Reichert, Bot. Jb. 56: 673 (1921)

Ref.: Reichert 1921, Melchers 1931.

36. Glonium salsolae Reichert, Bot. Jb. 56: 672 (1921)

Ref.: Reichert 1921, Melchers 1931.

Didymosphaeriaceae, Pleosporales

37. Didymosphaeria epidermidis (Fr.) Fuckel, Jb. nassau. Ver. Naturk. 23-24: 140 (1870)

Ref.: Reichert 1921, Melchers 1931.

Pleosporales genera, incertae sedis

38. Diplodina dracaenicola Sacc., Syll. fung. (Abellini) 3: 413 (1884)

Synonym:- Ascochyta dracaenicola (Sacc.) Allesch., Rabenh. Krypt.-Fl., Edn 2 (Leipzig) 1(6): 641 (1900)

Ref.: Reichert 1921.

39. Sporidesmium bicolor (S. Hughes) M.B. Ellis, Mycol. Pap. 70: 52 (1958)

Ref.: Melchers 1931.

40. Sporidesmium longipedicellatum Reichert, Bot. Jb. 56: 723 (1921)

Ref.: Reichert 1921. 
Massarinaceae, Pleosporales

41. Helminthosporium flexuosum Corda Icon. fung. (Prague) 1: 13 (1837)

Ref.: Thüemen 1878.

Phaeosphaeriaceae, Pleosporales

42. Ampelomyces quisqualis Ces., Bot. Ztg. 10: 301 (1852)

Ref.: Thüemen 1878, Melchers 1931.

43. Sphaerellopsis filum (Biv.) B. Sutton, Mycol. Pap. 141: 196 (1977)

Synonym:- Darluca filum (Biv.) Castagne, Outl. Brit. Fung. (London): 318 (1860)

Ref.: Reichert 1921.

44. Eudarluca caricis (Fr.) O.E. Erikss., Bot. Notiser 119: 33 (1966)

Ref.: Melchers 1931.

Pleosporaceae, Pleosporales

45. Alternaria brassicae (Berk.) Sacc., Michelia 2(no. 6): 129 (1880)

Ref.: Melchers 1931.

46. Alternaria citri Ellis \& N. Pierce, in Pierce, Bot. Gaz. 33: 234 (1902)

Ref.: Melchers 1931.

47. Alternaria cucumerina (Ellis \& Everh.) J.A. Elliott, Am. J. Bot. 4: 472 (1917)

Ref.: Melchers 1931.

48. Alternaria euphorbiicola E.G. Simmons \& Engelhard, in Simmons, Mycotaxon 25(1): 196 (1986)

Synonym:- Macrosporium euphorbiae Reichert, Bot. Jb. 56: 723 (1921)

Ref.: Reichert 1921, Melchers 1931.

49. Alternaria solani Sorauer, Z. PflKrankh. PflSchutz 6: 6 (1896)

Ref.: Melchers 1931.

50. Alternaria tomato (Cooke) L.R. Jones, Bull. Torrey bot. Club 23: 353 (1896) Ref.: Melchers 1931.

51. Bipolaris oryzae (Breda de Haan) Shoemaker, Can. J. Bot. 37 (5): 883 (1959)

Synonym:- Helminthosporium oryzae Breda de Haan, Bulletin Inst. Bot. Buitenzorg 6: 11 (1900)

Ref.: Melchers 1931.

52. Clathrococcum magnusianum Reichert, Bot. Jb. 56: 726 (1921)

Ref.: Reichert 1921, Melchers 1931.

53. Macrosporium oleae Reichert, Bot. Jb. 56: 724 (1921)

Ref.: Reichert 1921, Melchers 1931.

54. Pleospora asphodeli Rabenh., Un. Ital. Crypt. 12 (1866)

Ref.: Reichert 1921, Melchers 1931.

55. Pleospora egyptiaca Reichert [as 'aegyptiaca'], Bot. Jb. 56: 671 (1921)

Ref.: Reichert 1921, Melchers 1931.

56. Pleospora herbarum (Pers.) Rabenh., Klotzschii Herb. Viv. Mycol.: no. 547 (1854)

Synonym:- Macrosporium commune Rabenh., Fungi europ. exsicc.: no. 1360 (1870)

Ref.: Sickenberger 1901, Reichert 1921, Melchers 1931.

57. Pleospora lindaviana Reichert 1921, Bot. Jb. 56: 671 (1921)

Ref.: Reichert 1921, Melchers 1931.

58. Pleospora rotundata Reichert, Bot. Jb. 56: 671 (1921)

Ref.: Reichert 1921, Melchers 1931.

59. Pyrenophora graminea S. Ito \& Kurib., J. Fac. agric., Hokkaido Imp. Univ., Sapporo 29: 85-123 (1931)

Ref.: Melchers 1931.

60. Pyrenophora teres Drechsler, J. Agric. Res., Washington 24 (8): 656 (1923)

Ref.: Melchers 1931.

61. Setosphaeria turcica (Luttr.) K.J. Leonard \& Suggs, Mycologia 66(2): 295 (1974)

Synonym:- Helminthosporium turcicum Pass., Boln Comiz. Agr. Parmense 10: 3 (1876) 
Ref.: Melchers 1931.

Venturiaceae, Venturiales

62. Fusicladium artiges Pez. , in Sickenberger, Mémoires Présentés a L'Institut Égyptien 4(1): 331 (1900)

Ref.: Sickenberger 1901.

63. Fusicladium cynanchi Reichert, Bot. Jb. 56: 720 (1921)

Ref.: Reichert 1921, Melchers 1931.

Trichocomaceae, Eurotiales

\section{Ascomycota, Eurotiomycetes}

64. Aspergillus candidus Link, Mag. Gesell. naturf. Freunde, Berlin 3(1-2): 16 (1809)

Ref.: Reichert 1921, Melchers 1931.

65. Aspergillus niger Tiegh., Annls Sci. Nat., Bot., sér. 5 8: 240 (1867)

Synonym:- Ustilago phoenicis Corda, Icon. fung. (Prague) 4: 9 (1840)

Ref.: Thüemen 1878, Reichert 1921, Melchers 1931.

66. Aspergillus phoenicis Corda) Thom \& Currie, Journal of Agricultural Research 7: 9 (1916) Ref.: Reichert 1921.

67. Penicillium digitatum (Pers.) Sacc., Fungi italica autogr. del. 17-28: tab. 894 (1881)

Ref.: Melchers 1931.

68. Penicillium italicum Wehmer, Hedwigia 33: 211 (1894)

Ref.: Melchers 1931.

Ascomycota genera, incertae sedis

69. Bispora hammonis (Ehrenb.) Reichert, Bot. Jb. 56: 719 (1921)

Ref.: Reichert 1921, Melchers 1931.

70. Bispora opuntiicola Reichert, Bot. Jb. 56: 719 (1921)

Ref.: Reichert 1921, Melchers 1931.

71. Fumago vagans Pers., Mycol. eur. (Erlanga) 1: 9 (1822)

Ref.: Reichert 1921, Melchers 1931.

72. Hormiscium calligoni Reichert, Bot. Jb. 56: 718 (1921)

Ref.: Reichert 1921, Melchers 1931.

73. Hormiscium saccharicola Reichert, Bot. Jb. 56: 718 (1921)

Ref.: Reichert 1921, Melchers 1931.

Triblidiaceae, Triblidiales

74. Triblidium punctum (Pat.) Reichert [as 'Tryblidium'], Bot. Jb. 56: 673 (1921)

Basionym: Blitridium punctum Pat., Bull. Soc. mycol. Fr. 11: 87 (1895) interpreted as a new combination

Ref.: Patouillard 1895, Sickenberger 1901, Reichert 1921.

Erysiphaceae, Erysiphales

Ascomycota, Leotiomycetes

75. Blumeria graminis (DC.) Speer, Sydowia 27(21-26): 2 (1975)

Synonym:- Erysiphe graminis DC., in de Candolle \& Lamarck, Fl. franç., Edn 3 (Paris) 6: $106(1815)$

Ref.: Magnus 1910, Reichert 1921, Melchers 1931.

76. Erysiphe cruciferarum Opiz ex L. Junell, Svensk bot. Tidskr. 61(1): 217 (1967)

Synonym:- Erysiphe communis (Wallr.) Schltdl., Fl. berol. (Berlin) 2: 168 (1824)

Ref.: Melchers 1931.

77. Erysiphe lamprocarpa (Wallr.) Link, in Willdenow, Sp. pl., Edn 4 6(1): 108 (1824)

Ref.: Thüemen 1880.

78. Erysiphe necator Schwein., Trans. Am. phil. Soc., New Series 4(2): 270 (1832)

Ref.: Melchers 1931.

79. Erysiphe polygoni DC., Fl. agen. 2: 614 (1821)

Ref.: Reichert 1921. 
80. Fibroidium abelmoschi (Thüm.) U. Braun \& R.T.A. Cook, Taxonomic Manual of the Erysiphales (Powdery Mildews): 167 (2012)

Synonym:- Oidium abelmoschi Thüm., Grevillea 6(no. 39): 102 (1878)

Ref.: Thüemen 1878, Thüemen 1879, Thüemen 1880, Sickenberger 1901, Reichert 1921, Melchers 1931.

81. Golovinomyces cichoracearum (DC.) V.P. Heluta [as 'cichoraceorum'], Ukr. bot. Zh. 45(5): 62 (1988).

Synonym:- Erysiphe cichoracearum DC., in Lamarck \& de Candolle, Fl. franç., Edn 3 (Paris) 2: 274 (1805)

Ref.: Melchers 1931.

82. Leveillula taurica (Lév.) G. Arnaud, Annls Épiphyt. 7: 92 (1921) Ref.: Melchers 1931.

83. Oidium lippiae Thüm., Grevillea 6 (no. 39): 103 (1878) Ref.: Thüemen 1878, Reichert 1921, Melchers 1931.

84. Oidium mangiferae Berthet, Boln Agric., São Paulo 15: 818 (1914) Ref.: Melchers 1931.

85. Oidium medicagineum Thüm. Grevillea. 8:49 (1879)

Ref.: Thüemen 1879, Reichert 1921, Melchers 1931.

86. Podosphaera clandestina (Wallr.) Lév., Annls Sci. Nat., Bot., sér. 3 15: 136 (1851) Ref.: Melchers 1931.

87. Podosphaera fuliginea (Schltdl.) U. Braun \& S. Takam., Schlechtendalia 4: 29 (2000) Synonym:- Oidium erysiphoides Fr., Syst. mycol. (Lundae) 3(2): 432 (1832)

- Oidium erysiphoides Fr. f. cucrbitae maximae (1832)

Ref.: Thüemen 1878, Thüemen 1879, Thüemen 1880, Sickenberger 1901, Reichert 1921, Melchers 1931.

88. Podosphaera pannosa (Wallr.) de Bary, Abh. senckenb. naturforsch. Ges. 1(no. 3): 48 (1870)

Synonym:- Oidium leucoconium Desm., Pl. Crypt. Nord France, Edn 1: no. 303 (1846) -Sphaerotheca pannosa (Wallr.) Lév., Annls Sci. Nat., Bot., sér. 3 15: 138 (1851)

Ref.: Thüemen 1878, Reichert 1921, Melchers 1931.

\section{Dermateaceae, Helotiales}

89. Diplocarpon rosae F.A. Wolf, Bot. Gaz. 54: 231 (1912)

Ref.: Melchers 1931.

90. Gloeosporium schweinfurthianum Thüm., Grevillea VII., p. 49 (1879)

Ref.: Thüemen 1879, Reichert1921, Melchers 1931.

91. Marssonina kriegeriana (Bres.) Magnus, Hedwigia 45: 88 (1906)

Ref.: Reichert 1921, Melchers 1931.

92. Pseudopeziza medicaginis (Lib.) Sacc., Malpighia 1: 455 (1887)

Ref.: Melchers 1931.

93. Pseudopeziza trifolii (Biv.) Fuckel, Jb. nassau. Ver. Naturk. 23-24: 290 (1870)

Ref.: Melchers 1931.

Helotiales genera, incertae sedis

94. Coniothecium heterosporum Reichert, Bot. Jb. 56: 722 (1921)

Ref.: Reichert 1921, Melchers 1931.

95. Coniothecium tamariscinum Thüm., , Flora, Regensburg 63: 477 (1880)

Ref.: Thüemen 1880, Reichert 1921, Melchers 1931.

96. Coniothyrium diedickeanum Reichert, Bot. Jb. 56: 713 (1921)

Ref.: Reichert 1921, Melchers 1931.

97. Coniothyrium sporoboli Reichert, Bot. Jb. 56: 713 (1921)

Ref.: Reichert 1921, Melchers 1931. 
98. Monostichella salicis (Westend.) Arx, Verh. K. ned. Akad. Wet., tweede sect. 51(3): 131 (1957)

Synonym:- Gloeosporium salicis Westend., Pl. crypt. exsicc.: no. 1269 (1859)

Ref.: Melchers 1931.

Sclerotiniaceae, Helotiales,

99. Botrytis cinerea Pers., Ann. Bot. (Usteri) 1: 32 (1794)

Ref.: Reichert 1921, Melchers 1931.

Rhytismataceae, Rhytismatales

100. Leptostroma donacinum (Sacc.) Sacc., Michelia 2(no. 7): 352 (1881)

Ref.: Reichert 1921, Melchers 1931.

Morchellaceae, Pezizales

Ascomycota, Pezizomycetes

101. Morchella esculenta (L.) Pers., Syn. meth. fung. (Göttingen) 2: 618 (1801)

Synonym:- Morchella cónica Pers., Traité sur les Champignons Comestibles (Paris): 257 (1818)

-Morchella esculenta f. rotunda (Pers.) Reichert, Bot. Jb. 56: 673 (1921)

Ref.: Reichert 1921, Melchers 1931.

102. Terfezia arenaria (Moris) Trappe, Trans. Br. mycol. Soc. 57(1): 90 (1971)

Synonym:- Terfezia leonis (Tul. \& C. Tul.) Tul. \& C. Tul., Fungi hypog.: 173 (1851)

Ref.: Sickenberger 1901, Reichert 1921, Melchers 1931.

103. Terfezia deflersii Pat., J. Bot., Paris 8: 154 (1894)

Ref.: Sickenberger 1901, Reichert 1921, Melchers 1931.

104. Tirmania nivea (Desf.) Trappe, Trans. Br. mycol. Soc. 57(1): 88 (1971)

Synonym:- Tirmania africana Chatin, Truffe, Edn 2: 80 (1892)

- Tirmania ovalispora (Pat.) Pat., J. Bot., Paris 8: 155 (1894)

Ref.: Reichert 1921, Melchers 1931.

Diaporthaceae, Diaporthales

Ascomycota, Sordariomycetes

105. Phomopsis citri H.S. Fawc., Phytopathology 2(3): 109 (1912)

Ref.: Melchers 1931.

Melanconidaceae, Diaporthales

106. Melanconium echinosporum Reichert, Bot. Jb. 56: 715 (1921)

Ref.: Reichert 1921, Melchers 1931.

Hypocreaceae, Hypocreales

107. Hypomyces galericola Henn., Hedwigia 41: 214 (1902)

Ref.: Reichert 1921, Melchers 1931.

Incertae sedis, Hypocreales

108. Stilbella dielsiana Reichert, Bot. Jb. 56: 725 (1921)

Ref.: Reichert 1921, Melchers 1931.

109. Trichothecium roseum (Pers.) Link, Mag. Gesell. naturf. Freunde, Berlin 3(1-2): 18 (1809)

Synonym:- Cephalothecium roseum Corda, Icon. fung. (Prague) 2: 14 (1838)

Ref.: Melchers 1931.

Glomerellaceae, Incertae sedis

110. Colletotrichum gloeosporioides (Penz.) Penz. \& Sacc., Atti Inst. Veneto Sci. lett., ed Arti, Sér. 6 2: 670 (1884)

Ref.: Reichert 1921, Melchers 1931.

111. Vermicularia culmifraga Fr., Summa veg. Scand., Section Post. (Stockholm): 420 (1849)

Ref.: Magnus 1910, Reichert 1921, Melchers 1931.

Magnaporthaceae, Magnaporthales

112. Clasterosporium lindavianum Reichert, Bot. Jb. 56: 722 (1921) 
Ref.: Reichert 1921, Melchers 1931.

\section{Nectriaceae, Hypocreales}

113. Fusarium neocosmosporiellum O'Donnell \& Geiser, Phytopathology 103(5): 400408 (2013)

Synonym:- Neocosmospora vasinfecta E.F. Sm., Bull. U.S. Department of Agriculture 17: 45 (1899)

Ref.: Reichert 1921, Melchers 1931.

114. $\quad$ Fusarium uredinicola Jul. Müll., Ber. dt. bot. Ges. 3: 395 (1885)

Ref.: Reichert 1921, Melchers 1931.

115. Gibberella pulicaris (Kunze) Sacc., Michelia 1(no. 1): 43 (1877)

Synonym:- Fusarium roseum Link, Mag. Gesell. naturf. Freunde, Berlin 3(1-2): 10 (1809)

Ref.: Sickenberger 1901, Reichert 1921, Melchers 1931.

116. Haematonectria haematococca (Berk. \& Broome) Samuels \& Rossman, in Rossman, Samuels, Rogerson \& Lowen, Stud. Mycol. 42: 135 (1999)

Synonym:- Fusarium solani (Mart.) Sacc., Michelia 2(no. 7): 296 (1881)

Ref.: Melchers 1931.

Niessliaceae, Hypocreales

117. Melanopsamma pomiformis (Pers.) Sacc., Michelia 1(no. 3): 347 (1878)

Ref.: Reichert 1921, Melchers 1931.

Phyllachoraceae, Phyllachorales

118. Phyllachora cynodontis Niessl, Verh. nat. Ver. Brünn 14: 219 (1876)

Ref.: Thüemen 1878, Magnus 1910, Reichert 1921, Melchers 1931.

119. Phyllachora ehrenbergii Reichert, Bot. Jb. 56: 668 (1921)

Ref.: Reichert 1921, Melchers 1931.

120. Phyllachora graminis (Pers.) Fuckel, Jb. nassau. Ver. Naturk. 23-24: 216 (1870) Ref.: Melchers 1931.

121. Sphaerodothis schweinfurthii Reichert, Bot. Jb. 56: 669 (1921)

Ref.: Reichert 1921, Melchers 1931.

Trichosphaeriaceae, Trichosphaeriales

122. Brachysporium flexuosum (Corda) Sacc., Syll. fung. (Abellini) 4: 429 (1886)

Ref.: Reichert 1921, Melchers 1931.

Diatrypaceae, Xylariales

123. Eutypa hypoxantha (Lév.) Starbäck, Ascomy. Regn. Exped. Vet. Akad. Handl. 25(no. 3): 63 (1900)

Ref.: Melchers 1931.

Xylariaceae, Xylariales

124. Xylaria hypoxylon (L.) Grev., Fl. Edin.: 355 (1824)

Ref.: Reichert 1921, Melchers 1931.

Agaricaceae, Agaricales

\section{Basidiomycota, Agaricomycetes}

125. $\quad$ Agaricus campestris L. [as 'campester'], Sp. pl. 2: 1173 (1753)

Synonym:- Psalliota campestris (L.) Quél., Mém. Soc. Émul. Montbéliard, Sér. 2 5: 140 (1872)

Ref.: Decaisne 1835, Roumeguère 1887, Sickenberger 1901, Reichert 1921, Melchers 1931.

126. Battarrea phalloides (Dicks.) Pers., Syn. meth. fung. (Göttingen) 1: xiv, 129 (1801) Ref.: Reichert 1921, Melchers 1931.

127. Crucibulum laeve (Huds.) Kambly, Gast. Iowa: 167 (1936)

Synonym: -Crucibulum vulgare Tul. \& C. Tul., Annls Sci. Nat., Bot., sér. 3 1: 90 (1844)

Ref.: Melchers 1931.

128. Cyathus stercoreus (Schwein.) De Toni, in Berlese, De Toni \& Fischer, Syll. fung. (Abellini) 7: 40 (1888) 
Ref.: Reichert 1921, Melchers 1931.

129. Gyrophragmium delilei Mont., Annls Sci. Nat., Bot., sér. 2 20: 77 (1843)

Ref.: Sickenberger 1901, Magnus 1910, Reichert 1921, Melchers 1931.

130. Leucoagaricus leucothites (Vittad.) Wasser, Ukr. bot. Zh. 34(3): 308 (1977)

Synonym:- Lepiota holosericea Gillet, Hyménomycètes (Alençon): 67 (1874) [1878]

Ref.: Reichert 1921, Melchers 1931.

131. Leucoagaricus meleagris (Gray) Singer, Lilloa 22: 422 (1951) [1949]

Synonym:- Lepiota meleagris (Gray) Sacc., Syll. fung. (Abellini) 5: 36 (1887)

Ref.: Reichert 1921, Melchers 1931.

132. Montagnea candollei (Fr.) Fr., in Corda in Zobel, Icon. fung. (Prague) 6: 85 (1854)

Synonym:- Montagnites candollei Fr. Epicr. syst. mycol. (Upsaliae): 241 (1838) [18361838]

Ref.: Sickenberger 1901.

133. Montagnea radiosa (Pall.) Šebek, Česká Mykol. 8(3): 144 (1954)

Synonym:- Montagnites radiosus (Pall.) Henn., Hedwigia 40: 98 (1901).

Ref.: Reichert 1921, Melchers 1931.

134. Podaxis arabicus Pat. [as 'Podaxon'], Bull. Soc. Mycol. France 3: 122, 123. (1887)

Ref.: Sickenberger 1901, Reichert 1921, Melchers 1931.

135. $\quad$ Podaxis calyptratus Fr. Syst. mycol. (Lundae) 3(1): 63 (1829)

Ref.: Reichert 1921, Melchers 1931.

136. Podaxis carcinomalis (L. f.) Fr. Syst. mycol. (Lundae) 3(1): 62 (1829)

Ref.: Reichert 1921, Melchers 1931.

137. Podaxis deflersii Pat. Bull. Soc. Mycol. France 6: 165 (1890)

Ref.: Reichert 1921, Melchers 1931.

138. Podaxis egyptiacus Mont. Annls Sci. Nat., Bot., sér. 2 4: 195 (1835)

Ref.: Decaisne 1835, Reichert 1921, Melchers 1931.

139. Podaxis indicus (Spreng.) Massee, J. Bot., Lond. 28: 74 (1890)

Ref.: Reichert 1921, Melchers 1931.

140. Podaxis Squamosus Pat. Bull. Soc. Mycol. France 13: 209 (1891)

Ref.: Sickenberger 1901, Reichert 1921, Melchers 1931.

141. Podaxon ovatus Rosm. Mémoires Présentés a L'Institut Égyptien 4(1): 332 (1900)

Ref.: Sickenberger 1901.

142. Tulostoma boissieri Kalchbr.,in Roumeguère, Revue mycol., Toulouse 3: 24 (1881)

Ref.: Roumeguère 1881, Sickenberger 1901.

143. Tulostoma brumale Pers., Neues Mag. Bot. 1: 86 (1794)

Synonym:- Tulostoma mammosum P. Micheli ex Fr., Syst. mycol. (Lundae) 3(1): 42 (1829)

Ref.: Reichert 1921, Melchers 1931.

144. Tulostoma tortuosum Ehrenb., Horae Phys. Berol. (1820)

Ref.: Reichert 1921.

Bolbitiaceae, Agaricales

145. Conocybe tenera (Schaeff.) Fayod, Annls Sci. Nat., Bot., sér. 7 9: 357 (1889)

Ref.: Melchers 1931.

\section{Cortinariaceae, Agaricales}

146. Cortinarius bolaris (Pers.) Fr., Epicr. syst. mycol. (Upsaliae): 282 (1838)

Synonym:- Inoloma bolare bolare (Pers.) Wünsche, Die Pilze: 127 (1877)

Ref.: Reichert 1921.

147. Galera teñera (Schaeff.) P. Kumm., Führ. Pilzk. (Zerbst): 75 (1871)

Ref.: Reichert 1921.

148. Galerina vittiformis (Fr.) Singer, Acta Inst. bot. Komarov. Acad. Sci., Pl. Crypt, ser.

2 6: 472 (1950)

Synonym:- Galera rubiginosa (Pers.) Fr., Bidr. Känn. Finl. Nat. Folk 1: 440 (1879)

Ref.: Reichert 1921 - Melchers 1931. 


\section{Incertae sedis, Agaricales}

149. Panaeolus fimicola (Pers.) Gillet, Hyménomycètes (Alençon): 621 (1878) Ref.: Reichert 1921, Melchers 1931.

150. Panaeolus papilionaceus (Bull.) Quél., Mém. Soc. Émul. Montbéliard, Sér. 2 5: 152 [122 repr.] (1872)

Synonym:- Panaeolus campanulatus (L.) Quél., Mém. Soc. Émul. Montbéliard, Sér. 2 5: $151(1872)$

Ref.: Reichert 1921- Melchers 1931.

151. Panaeolus retirugus (Fr.) Gillet, Hyménomycètes (Alençon): 621 (1878)

Synonym:- Panaeolus papilionaceus (Bull.) Quél., Mém. Soc. Émul. Montbéliard, Sér. 25: 152 [122 repr.] (1872)

Ref.: Reichert 1921.

152. Pilosace algeriensis (Fr.) Quél., Mém. Soc. Émul. Montbéliard, Sér. 2 5: 360 (1873)

Ref.: Roumeguère 1887, Sickenberger 1901, Reichert 1921, Melchers 1931.

\section{Phelloriniaceae, Agaricales}

153. Dictyocephalos attenuatus (Peck) Long \& Plunkett, Mycologia 32(6): 697 (1940)

Synonym:- Battarreopsis artinii Henn., Hedwigia 41(Beibl.): (212) (1902)

Ref.: Reichert 1921- Melchers 1931.

154. Phellorinia herculeana (Pers.) Kreisel, Česká Mykol. 15(4): 196 (1961)

Synonym:- Phellorinia delestrei (Durieu \& Mont.) E. Fisch., Nat. Pflanzenfam., Teil. I (Leipzig) $1^{* * *}: 334$ (1899)

Ref.: Roumeguère 1887- Reichert 1921- Melchers 1931.

\section{Physalacriaceae, Agaricales}

155. Armillaria mellea (Vahl) P. Kumm., Führ. Pilzk. (Zerbst): 134 (1871)

Synonym:- Clitocybe mellea (Vahl) Ricken, Die Blätterpilze: 362 (1915)

Ref.: Reichert 1921, Melchers 1931.

\section{Pleurotaceae, Agaricales}

156. Pleurotus foliicola Pat. \& Lagerh., Bull. Soc. mycol. Fr. 9: 127 (1893)

Ref.: Reichert 1921, Melchers 1931.

157. Pterophyllus bovei Lév., Annls Sci. Nat., Bot., sér. 3 2: 178 (1844)

\section{Pluteaceae, Agaricales}

Ref.: Melchers 1931.

158. Volvopluteus gloiocephalus (DC.) Vizzini, Contu \& Justo, in Justo, Vizzini, Minnis,

Menolli, Capelari, Rodríguez, Malysheva, Contu, Ghignone \& Hibbett, Fungal Biology 115(1): 15 (2011)

Synonym:- Volvaria speciosa (Fr.) P. Kumm., Führ. Pilzk. (Zerbst): 99 (1871)

Ref.: Reichert 1921- Melchers 1931.

\section{Psathyrellaceae, Agaricales}

159. Coprinellus disseminatus (Pers.) J.E. Lange Dansk bot. Ark. 9(no. 6): 93 (1938)

Synonym:- Agaricus disseminatus Pers., Syn. meth. fung. (Göttingen) 2: 403 (1801)

- Coprinus disseminatus (Pers.) Gray, Nat. Arr. Brit. Pl. (London) 1: 634 (1821)

Ref.: Reichert 1921, Melchers 1931.

160. Coprinellus micaceus (Bull.) Vilgalys, Hopple \& Jacq. Johnson, in Redhead,

Vilgalys, Moncalvo, Johnson \& Hopple, Taxon 50(1): 234 (2001)

Synonym:- Coprinus micaceus (Bull.) Fr., Epicr. syst. mycol. (Upsaliae): 247 (1838) [1836-1838]

Ref.: Reichert 1921, Melchers 1931.

161. Coprinopsis atramentaria (Bull.) Redhead, Vilgalys \& Moncalvo, in Redhead,

Vilgalys, Moncalvo, Johnson \& Hopple, Taxon 50(1): 226 (2001)

Synonym:- Coprinus atramentarius (Bull.) Fr., Epicr. syst. mycol. (Upsaliae): 243

(1838) [1836-1838] 
Ref.: Reichert 1921, Melchers 1931.

162. Coprinus barbeyi Kalchbr., Revue mycol., Toulouse 3: 24 (1881)

Ref.: Roumeguère 1881, Sickenberger 1901, Reichert 1921, Melchers 1931.

163. Coprinus clavatus Fr., Epicr. syst. mycol. (Upsaliae): 242 (1838)

Ref.: Roumeguère 1887, Reichert 1921, Melchers 1931.

164. Coprinus clavatus f. arenosa Roum., Revue mycol., Toulouse 9 (no. 36): 206 (1887)

Ref.: Reichert 1921.

165. Coprinus comatus (O.F. Müll.) Pers., Tent. disp. meth. fung. (Lipsiae): 62 (1797)

Ref.: Roumeguère 1887, Reichert 1921, Melchers 1931.

166. Coprinus comatus f. barbeyi Roum., Revue mycol., Toulouse 9(no. 36): 206 (1887)

Ref.: Reichert 1921.

167. Coprinus jasmundianus Kalchbr., Aschers. Beitr. Flora Aeg.: 73 (1879)

Ref.: Ascherson 1879, Thüemen 1879, Reichert 1921, Melchers 1931.

168. Coprinus sterquilinus (Fr.) Fr., Epicr. syst. mycol. (Upsaliae): 242 (1838)

Ref.: Reichert 1921, Melchers 1931.

169. Corynespora torulosa (Syd.) Crous, Persoonia, Mol. Phyl. Evol. Fungi 31: 211

(2013)

Synonym:- Cercospora musarum S.F. Ashby, Bull. Dept. Agric. (Kingston) 2: 109 (1913)

Ref.: Melchers 1931.

170. Psathyrella candolleana (Fr.) Maire, in Maire \& Werner, Mém. Soc. Sci. Nat. Maroc. 45: 112 (1937)

Synonym:- Hypholoma appendiculatum (Bull.) Quél., Mém. Soc. Émul. Montbéliard, Sér. 2 5: 146 (1872)

Ref.: Roumeguère 1887, Sickenberger 1901, Melchers 1931.

171. Psathyrella schweinfurthii Roum., Revue mycol., Toulouse 9: 205 (1887)

Ref.: Roumeguère 1887, Sickenberger 1901, Reichert 1921, Melchers 1931.

Schizophyllaceae, Agaricales

172. Schizophyllum commune Fr. Observ. mycol. (Havniae) 1: 103 (1815)

Ref.: Reichert 1921, Melchers 1931.

\section{Strophariaceae, Agaricales}

173. Agrocybe pediades (Fr.) Fayod, Annls Sci. Nat., Bot., sér. 7 9: 358 (1889)

Synonym:- Naucoria pediades (Fr.) P. Kumm., Führ. Pilzk. (Zerbst): 78 (1871)

Ref.: Reichert 1921, Melchers 1931.

174. Agrocybe vervacti (Fr.) Singer, Beih. Botan. Centralbl., Abt. B 56: 167 (1936)

Synonym:- Naucoria vervacti (Fr.) Quél., Mém. Soc. Émul. Montbéliard, Sér. 2 5: 132 (1872)

Ref.: Roumeguère 1881, Melchers 1931.

175. Flammula acuminatospora Reichert, Bot. Jb. 56: 703 (1921)

Ref.: Reichert 1921, Melchers 1931.

176. Flammula schweinfurthii Reichert, Bot. Jb. 56: 703 (1921)

Ref.: Reichert 1921, Melchers 1931.

177. Pholiota alexandrina Reichert, Bot. Jb. 56: 703 (1921)

Ref.: Reichert 1921, Melchers 1931.

\section{Tricholomataceae, Agaricales}

178. Clitocybe mairei Reichert, Bot. Jb. 56: 707 (1921)

Ref.: Reichert 1921, Melchers 1931.

179. Melanoleuca melaleuca (Pers.) Murrill, Mycologia 3(3): 167 (1911)

Synonym:- Tricholoma melaleucum (Pers.) P. Kumm., Führ. Pilzk. (Zerbst): 133 (1871)

Ref.: Reichert 1921, Melchers 1931. 
Atheliaceae, Atheliales

180. Athelia rolfsii (Curzi) C.C. Tu \& Kimbr., Bot. Gaz. 139(4): 460 (1978)

Synonym:- Sclerotium rolfsii Sacc., Annls mycol. 9(3): 257 (1911)

Ref.: Melchers 1931.

181. Byssoporia terrestris (DC.) M.J. Larsen \& Zak, Can. J. Bot. 56: 1123 (1978)

Synonym:- Poria terrestris Pers., Icon. Desc. Fung. Min. Cognit. (Leipzig) 3: 35 (1805)

Ref.: Reichert 1921.

Boletaceae, Boletales

182. Boletus subtomentosus L., Sp. pl. 2: 1178 (1753)

Ref.: Reichert 1921, Melchers 1931.

Coniophoraceae, Boletales

183. Gyrodontium sacchari (Spreng.) Hjortstam, Mycotaxon 54: 186 (1995)

Synonym:- Hydnum boveanum Mont., in Decaisne, Annls Sci. Nat., Bot., sér. 24: 194 (1835)

Ref.: Decaisne 1835, Reichert 1921, Melchers 1931.

\section{Suillaceae, Boletales}

184. Suillus bovinus (L.) Roussel, F. Calvados: 34 (1796)

Synonym:- Boletus bovinus L., Sp. pl. 2: 1177 (1753)

Ref.: Reichert 1921, Melchers 1931.

Botryobasidiaceae, Cantharellales

185. Botryobasidium vagum (Berk. \& M.A. Curtis) D.P. Rogers, Univ. Iowa Stud. nat. Hist. 17(1): 17 (1935)

Synonym:- Corticium vagum Berk. \& M.A. Curtis, Grevillea 1(no. 12): 179 (1873)

Ref.: Melchers 1931.

Fomitopsidaceae, Polyporales

186. Ceriomyces fici (Pat.) Sacc., Syll. fung. (Abellini) 14(1): 194 (1899)

Ref.: Reichert 1921, Melchers 1931.

187. Ptychogaster fici Pat., Expl. sci. Tunisie 4: 4 (1892)

Ref.: Sickenberger 1901.

Ganodermataceae, Polyporales

188. Ganoderma lucidum (Curtis) P. Karst., Revue mycol., Toulouse 3(no. 9): 17 (1881)

Synonym:- Fomes lucidus (Curtis) Sacc., Syll. fung. (Abellini) 6: 157 (1888)

Ref.: Reichert 1921, Melchers 1931.

\section{Gloeophyllaceae, Gloeophyllales}

189. Gloeophyllum odoratum (Wulfen) Imazeki, Bull. Tokyo Sci. Mus. 6: 75 (1943)

Synonym:- Trametes odorata (Wulfen) Fr., Epicr. syst. mycol. (Upsaliae): 489 (1838) [1836-1838]

Ref.: Reichert 1921, Melchers 1931.

Hymenochaetaceae, Hymenochaetales

190. Inonotus hispidus (Bull.) P. Karst., Meddn Soc. Fauna Flora fenn. 5: 39 (1879)

Synonym:- Polyporus hispidus (Bull.) Fr., Observ. mycol. (Havniae) 2: 260 (1818)

Ref.: Reichert 1921, Melchers 1931.

\section{Phallaceae, Phallales}

191. Dictyophora phalloidea Desv., J. Bot. (Desvaux) 2: 88 (1809)

Ref.: Reichert 1921, Melchers 1931.

192. Itajahya rosea (Delile) E. Fisch., Ber. dt. bot. Ges. 47(5): 294 (1929)

Synonym:- Phallus roseus Delile, Descript. de l'Egypte: 300 (1813)

Ref.: Delile 1813.

193. Phallus impudicus L., Sp. pl. 2: 1178 (1753)

Synonym:- Ithyphallus impudicus (L.) Fr., Syst. mycol. (Lundae) 2(2): 283 (1823)

Ref.: Reichert 1921, Melchers 1931. 
Polyporaceae, Polyporales

194. Fomes fomentarius (L.) Fr., Summa veg. Scand., Section Post. (Stockholm): 321 (1849)

Ref.: Reichert 1921, Melchers 1931.

195. Lentinus integrus Reichert, Bot. Jb. 56: 702 (1921)

Ref.: Reichert 1921, Melchers 1931.

196. Lentinus omphalopsis Reichert, Bot. Jb. 56: 702 (1921)

Ref.: Reichert 1921, Melchers 1931.

197. Neolentinus lepideus (Fr.) Redhead \& Ginns, Trans. Mycol. Soc. Japan 26(3): 357 (1985)

Synonym:- Lentinus lepideus (Fr.) Fr., Epicr. syst. mycol. (Upsaliae): 390 (1838) [18361838]

Ref.: Reichert 1921, Melchers 1931.

Stereaceae, Russulales

198. Stereum hirsutum (Willd.) Pers., Observ. mycol. (Lipsiae) 2: 90 (1800)

Ref.: Reichert 1921, Melchers 1931.

Entylomataceae, Entylomatales

Basidiomycota, Exobasidiomycetes

199. Entyloma schweinfurthii Henn., Hedw.: 210 (1902)

Ref.: Reichert 1921, Melchers 1931.

Graphiolaceae, Exobasidiales

200. Graphiola phoenicis (Moug. ex Fr.) Poit., Annls Sci. Nat., Bot., sér. 1 3: 473 (1824)

Ref.: Sickenberger 1901, Magnus 1910, Reichert 1921, Melchers 1931.

Georgefischeriaceae, Georgefischeriales

201. Jamesdicksonia linearis (Berk. \& Broome) Vánky, Mycotaxon 91: 259 (2005)

Synonym:- Uromyces linearis Berk. \& Broome, J. Linn. Soc., Bot. 14(no. 74): 92 (1873) [1875]

Ref.: Reichert 1921, Melchers 1931.

Microbotryaceae, Microbotryales

Basidiomycota, Microbotryomycetes

202. Sphacelotheca danthoniae (Kalchbr.) Reichert, Bot. Jb. 56: 681 (1921)

Ref.: Reichert 1921.

203. Sphacelotheca ischaemi (Fuckel) G.P. Clinton, J. Mycol. 8(3): 140 (1902)

Synoym:- Ustilago ischaemi Fuckel, Jb. nassau. Ver. Naturk. 15: 22 (1860)

Ref.: Thüemen 1880, Magnus 1910, Reichert 1921, Melchers 1931.

204. Sphacelotheca reiliana (J.G. Kühn) G.P. Clinton, J. Mycol. 8(3): 141 (1902)

Ref.: Reichert 1921, Melchers 1931.

205. Sphacelotheca schweinfurthiana (Thüm.) Sacc., Annls mycol. 6(6): 554 (1908)

Synonym:- Ustilago schweinfurthiana Thüm., Mycoth. Univ., cent. 8: no. 726 (1877)

Ref.: Thüemen 1878, Ascherson 1879, Sickenberger 1901,Magnus 1910.

Tilletiaceae, Tilletiales

206. Tilletia caries (DC.) Tul. \& C. Tul., Annls Sci. Nat., Bot., sér. 3 7: 113 (1847)

Synonym:- Tilletia tritici (Bjerk.) G. Winter, Die Brand des Getreide: 13 (1874)

Ref.: Reichert 1921, Melchers 1931.

207. Tilletia laevis J.G. Kühn, in Rabenhorst, Rabenhorst's Fungi europ. exsicc.: no. 1697 (1873)

Ref.: Melchers 1931.

Incertae sedis, Pucciniales

Basidiomycota, Pucciniomycetes

208. Aecidium cressae DC., in de Candolle \& Lamarck, Fl. franç., Edn 3 (Paris) 6: 89 (1815)

Ref.: Thüemen 1878, Roumeguère 1881. 
209. Aecidium suaedae Thüm., Flora, Regensburg 63: 478 (1880)

Ref.: Thüemen 1880.

210. Uredo aviculariae Alb. \& Schwein. ex Thüm., Flora, Regensburg 63: 478 (1880)

Ref.: Thüemen 1880.

211. Uredo coloni Reichert, Bot. Jb. 56: 696 (1921)

Ref.: Reichert 1921, Melchers 1931.

212. Uredo cyperi-alopecuroidis Reichert, Bot. Jb. 56: 697 (1921)

Ref.: Reichert 1921, Melchers 1931.

213. Uredo cypericola Henn., in Engler, Pflanzenw. Ost-Afrikas Nachbarg., Teil C: 52 (1895)

Ref.: Melchers 1931.

214. Uredo danthoniae Henn., Hedwigia 41: 211 (1902)

Ref.: Reichert 1921, Melchers 1931.

215. Uredo reaumuriicola Henn., Hedwigia 39: (154) (1900)

Ref. Reichert 1921.

216. Uredo euphorbiae-prunifoliae Reichert, Bot. It. 56: 697 (1921)

Ref.: Reichert 1921, Melchers 1931.

217. Uredo ficus Ravenel, F. Am. Exs., n. 485, Hoffm. Ind. P. 146

Ref. Thüemen 1880.

218. Uredo frankeniae Mont., in Webb \& Berthelot, Hist. nat. Iles Canar. (Paris) 3(2): 90 (1840)

Ref. Thüemen 1879.

219. Uredo linearis f. hordei-vulgaris Thüm., Grevillea 6(no. 39): 104 (1878)

Ref. Thüemen 1878.

220. Uredo reaumuriicola Henn., Hedwigia 39: 154 (1900)

Ref : Melchers 1931.

221. Uredo schanginiae Thüm., Grevillea 6(no. 39): 103 (1878)

Ref.: Thüemen 1878, Thüemen 1880.

222. Uredo zygophylli Henn., Bull. Herb. Boissier 1: 113 (1893)

Ref: Reichert 1921, Melchers 1931.

\section{Melampsoraceae, Pucciniales}

223. Melampsora euphorbiae (Ficinus \& C. Schub.) Castagne, Observ. Uréd. 2: 18

(1843)

Synonym:- Melampsora euphorbiae-gerardianae W. Muell., Centbl. Bakt. ParasitKde, Abt. I 2: 548 (1907)

-Melampsora euphorbiae f. euphorbiae-peplidis Thüm., Grevillea 8(no. 46): 51 (1879)

- Melampsora euphorbiae Cast. f. euphorbice prunifolice Thüm., Grevillea 8(no. 46): 51 (1879).

- Melampsora helioscopiae (Pers.) G. Winter, Rabenh. Krypt.-Fl., Edn 2 (Leipzig) 1.1: 240 (1881) [1884].

- Melampsora ricini Pass. ex E.A. Noronha, Agron. lusit. 14: 229, 242 (1952)

Ref.: Thüemen 1879, Roumeguère 1881, Reichert 1921, Melchers 1931.

224. Melampsora lini-usitatissimi Kuprev., in Kuprevicz \& Tranzschel, Flora Plant.

Crypt. URSS, 4, Fungi (1), 374 (1957)

Synonym: - Melampsora lini. Tul. f. lini-usitatissimi Thüm., Grevillea 6(no. 39): 104 (1878)

Ref.: Thüemen 1878.

225. Melampsora lini (Ehrenb.) Lév., Annls Sci. Nat., Bot., sér. 3 8: 376 (1847)

Ref.: Thüemen 1879, Reichert 1921, Melchers 1931. 
226. Melampsora salicis-albae Kleb., Pringsheims Jb. Wissenschaftl. Botanik 35: 679 (1901)

Ref.: Melchers 1931.

\section{Phakopsoraceae, Pucciniale}

227. Cerotelium fici (Castagne) Arthur, Bull. Torrey bot. Club 44: 509 (1917)

Synonym:- Kueneola fici E.J. Butler, Annls mycol. 12(2): 79 (1914)

Ref.: Reichert 1921, Melchers 1931.

\section{Phragmidiaceae, Pucciniales}

228. Phragmidium mucronatum (Pers.) Schltdl., Fl. berol. (Berlin) 2: 156 (1824)

Synonym:- Phragmidium disciflorum (Tode) J. James, Contr. U.S. natnl. Herb. 3(4): 276 (1895)

- Phragmidium subcorticium (Schrank) G. Winter, Rabenh. Krypt.-Fl., Edn 2 (Leipzig) 1.1: 228 (1881) [1884]

- Phragmidium rosarum Fuckel, Jb. nassau. Ver. Naturk. 23-24: 47 (1870) [1869-70]

Ref.: Thüemen 1878, Reichert 1921, Melchers 1931.

229. Phragmidium violaceum (Schultz) G. Winter, Hedwigia 19: 54 (1880)

Ref.: Melchers 1931.

Pucciniaceae, Pucciniales

230. Puccinia aristidae Tracy, J. Mycol. 7(3): 281 (1893)

Ref.: Reichert 1921, Melchers 1931.

231. Puccinia aristidicola Henn., Hedwigia 35(5): 243 (1896)

Ref.: Reichert 1921.

232. Puccinia asphodeli Moug., in Duby, Bot. Gall., Edn 2 (Paris) 2: 891 (1830)

Ref.: Reichert 1921, Melchers 1931.

233. Puccinia barbeyi (Roum.) Magnus, Bot. Ztg. 41: 115 (1883)

Ref.: Roumeguère 1881, Melchers 1931.

234. Puccinia calcitrapae DC., in Lamarck \& de Candolle, Fl. franç., Edn 3 (Paris) 2: 221 (1805)

Ref.: Reichert 1921, Melchers 1931.

235. Puccinia caricina DC., in de Candolle \& Lamarck, Fl. franç., Edn 3 (Paris) 5/6: 60 (1815)

Ref. Melchers 1931.

236. Puccinia carthami Corda, Icon. fung. (Prague) 4: 15 (1840)

Ref. Sickenberger 1901, Reichert 1921, Melchers 1931.

237. Puccinia carthami f. carthami Corda, Icon. fung. (Prague) 4: 15 (1840)

Ref. Melchers 1931.

238. Puccinia centaureae Mart., Fl. franç., Edn 3 (Paris) 6: 59 (1815)

Ref. Reichert 1921.

239. Puccinia cesatii J. Schröt., in Cohn, Beitr. Biol. Pfl. 3: 70 (1879)

Ref. Magnus 1910, Reichert 1921, Melchers 1931.

240. Puccinia chrysanthemi Roze, Bull. Soc. mycol. Fr. 16: 92 (1900)

Synonym: -Puccinia absinthii DC., Encycl. Méth. Bot. 8: 245 (1808)

Ref.: Reichert 1921, Melchers 1931.

241. Puccinia coronifera Kleb., Z. PflKrankh. PflSchutz 4: 135 (1894)

Ref. Reichert 1921, Melchers 1931.

242. Puccinia cressae Lagerh., Bolm Soc. broteriana, Coimbra, sér. 1 7: 131 (1889)

Ref. Reichert 1921, Melchers 1931.

243. Puccinia cynosuroides (Henn.) Syd., Annls mycol. 5(6): 494 (1907)

Ref. Reichert 1921, Melchers 1931.

244. Puccinia dioicae Magnus, Amt. Ber. 50 Versammt. D. Naturf. Ärzte München: 199 (1877) 
Synonym:- Puccinia caricis Rebent., Prodr. fl. neomarch. (Berolini): 356 (1804)

Ref. Magnus 1910, Reichert 1921, Melchers 1931.

245. Puccinia eryngii DC., Encycl. Méth. Bot. 8: 249 (1808)

Ref. Reichert 1921, Melchers 1931.

246. Puccinia frankeniae Link, Mag. Gesell. naturf. Freunde, Berlin 8: 30 (1816)

Ref. Reichert 1921, Melchers 1931.

247. Puccinia glumarum J.C. Schmidt, Mykologische Hefte (Leipzig) 1: 72 (1817)

Ref. Reichert 1921.

248. Puccinia graminis Pers., Neues Mag. Bot. 1: 119 (1794)

Ref. Reichert 1921, Melchers 1931.

249. Puccinia helianthi Schwein., Schr. naturf. Ges. Leipzig 1: 73 (1822)

Ref. Melchers 1931.

250. Puccinia hordei G.H. Otth, Mitt. naturf. Ges. Bern 711-744: 114 (1871) [1870]

Synonym: Puccinia simplex (Körn.) Erikss. \& Henning, Z. PflKrankh. PflSchutz 4: 260 (1894)

Ref. Reichert 1921, Melchers 1931.

251. Puccinia isiacae (Thüm.) G. Winter, in Kunze, Plantae orient-ross.: 127 (1887)

Synonym:- Uredo isiacae Thüm., in Schweinfurth \& Thümen, Grevillea 8(no. 46): 50 (1879)

Ref.: Thüemen 1879, Reichert 1921, Melchers 1931.

252. Puccinia launaeae Maire, Bull. Soc. bot. Fr. 54: CCIII (1907)

Ref. Magnus 1910, Reichert 1921, Melchers 1931.

253. Puccinia longissima J. Schröt., in Cohn, Beitr. Biol. Pfl. 3: 70 (1879)

Ref. Reichert 1921, Melchers 1931.

254. Puccinia magnusiana Körn., Hedwigia 15: 179 (1876)

Ref. Reichert 1921, Melchers 1931.

255. Puccinia menthae Pers., Syn. meth. fung. (Göttingen) 1: 227 (1801)

Ref: Melchers 1931.

256. Puccinia paraphysaria Bagnis, Atti R. Acad. Lincei, Mem. Cl. Sci. Fis., Ser. Sec. 3(2): 713 (1877)

Ref: Roumeguère 1881.

257. Puccinia paraphysata Reichert, Bot. Jb. 56: 96 (1921)

Ref. Reichert 1921, Melchers 1931.

258. Puccinia pimpinellae (F. Strauss) Link, in Willdenow, Sp. pl., Edn 4 6(1): 77 (1824)

Ref. Sickenberger 1901.

259. Puccinia porri (Sowerby) G. Winter, Rabenh. Krypt.-Fl., Edn 2 (Leipzig) 1.1: 200 (1881)

Ref. Melchers 1931.

260. Puccinia pulvinata Rabenh., Hedwigia 10: 20 (1871)

Ref. Magnus 1910, Reichert 1921, Melchers 1931.

261. Puccinia purpurea Cooke, Grevillea 5(no. 33): 15 (1876)

Ref. Reichert 1921, Melchers 1931.

262. Puccinia recondita Dietel \& Holw., Bull. Soc. bot. Fr. 4: 798 (1857)

Synonym:- Puccinia bromina Erikss., Annls Sci. Nat., Bot., sér. 8 9: 271 (1899)

- Puccinia dispersa Erikss. \& Henning, Bull. Inst. bot. Univ. Belgrade 12: 315 (1894)

- Puccinia triticina Erikss., Annls Sci. Nat., Bot., sér. 8 9: 270 (1899)

Ref.: Sickenberger 1901, Magnus 1910,Reichert 1921, Melchers 1931.

263. Puccinia rimosa (Link) G. Winter, Hedwigia 19: 28 (1880)

Ref. Reichert 1921, Melchers 1931.

264. Puccinia rottboelliae P. Syd. \& Syd., Monogr. Uredin. (Lipsiae) 1(5): 800 (1904)

Ref. Reichert 1921, Melchers 1931. 
265. Puccinia rufipes Dietel, Bot. Jb. 32: 48 (1903)

Ref. Magnus 1910, Melchers 1931.

266. Puccinia santolinae Magnus, Hedwigia 49: 97 (1908)

Ref. Magnus 1910, Reichert 1921, Melchers 1931.

267. Puccinia scirpi DC., in Lamarck \& de Candolle, Fl. franç., Edn 3 (Paris) 2: 223 (1805)

Ref. Reichert 1921, Melchers 1931.

268. Puccinia sorghi Schwein., Trans. Am. phil. Soc., New Series 4(2): 295 (1832)

Ref.: Melchers 1931.

269. Puccinia striiformis Westend., Bull. Acad. R. Sci. Belg., Cl. Sci. 21(2): 235 (1854) Ref.: Melchers 1931.

270. Puccinia turgida P. Syd. \& Syd., Monogr. Uredin. (Lipsiae) 1(2): 266 (1902)

Ref.: Melchers 1931.

271. Puccinia verruca Thüm., Revue mycol., Toulouse 1: 9 (1879)

Ref.: Thüiemen 1879, Reichert 1921, Melchers 1931.

272. Uromyces anthyllidis (Grev.) J. Schröt.,Hedwigia 14: 162 (1875)

Ref.: Reichert 1921, Melchers 1931.

273. Uromyces appendiculatus F. Strauss, Exanth. Pflanzen. (Wien): 277 (1833)

Ref.: Melchers 1931.

274. Uromyces chenopodii (Duby) J. Schröt., in Kunze, Fung. sel. exs., cent. 3: no. 214 (1880)

Ref.: Reichert 1921, Melchers 1931.

275. Uromyces lineolatus (Desm.) J. Schröt., in Rabenhorst, Fungi europ. exsicc.: no. 2077 (1876)

Synonym:- Uromyces scirpi Burrill, Bot. Gaz. 9: 188 (1884)

Ref.: Reichert 1921, Melchers 1931.

276. Uromyces muscari Lév., Annls Sci. Nat., Bot., sér. 3 8: 376 (1847)

Synonym:- Uromyces scillarum (Grev.) G. Winter, Pilze Deutschl.: 142 (1884)

Ref.: Roumeguère 1881, Reichert 1921, Melchers 1931.

277. Uromyces pisi-sativi (Pers.) Liro, Bidr. Känn. Finl. Nat. Folk 65: 100 (1908)

Synonym:- Aecidium euphorbiae Pers., in Gmelin, Systema Naturae, Edn 13 2(2): 1472 (1792)

- Uromyces astragali (Opiz) Sacc., Mycotheca veneti: no. 208 (1873)

Ref.: Roumeguère 1881, Reichert 1921, Melchers 1931.

278. Uromyces polygoni-avicularis (Pers.) G.H. Otth, Mitt. naturf. Ges. Bern 531-552: 87 (1864)

Synonym:- Uromyces polygoni (Pers.) Fuckel, Jb. nassau. Ver. Naturk. 23-24: 64 (1870) [1869-70]

Ref.: Magnus 1910, Reichert 1921, Melchers 1931.

279. Uromyces prö̈minens (DC.) Lév., Annls Sci. Nat., Bot., sér. 3 8: 371 (1847)

Ref.: Melchers 1931.

280. Uromyces renovatus P. Syd. \& Syd., Monogr. Uredin. (Lipsiae) 2(1): 113 (1909)

Ref.: Reichert 1921, Melchers 1931.

281. Uromyces rumicis (Schumach.) G. Winter, Pilze Deutschl.: 145 (1884)

Ref.: Reichert 1921, Melchers 1931.

282. Uromyces rumicum Lév., Annls Sci. Nat., Bot., sér. 3 8: 371 (1847)

Ref.: Thüemen 1880.

283. Uromyces schanginiae Thüm., Grevillea 6(no. 39): 103 (1878)

Ref.: Thüemen 1878, Reichert 1921, Melchers 1931.

284. Uromyces setariae-italicae Yoshino, Bot. Mag., Tokyo 20: 247 (1906)

Ref.: Melchers 1931. 
285. Uromyces striatus J. Schröt., Abh. Schles. Ges. Vaterl. Kult. Abth. Naturwiss. 48: $11(1870)$

Ref.: Thüemen 1880, Reichert 1921, Melchers 1931.

286. Uromyces trigonellae Pass., in Thümen, Herb. myc. oeconom. 3: no. 118 (1873)

Ref.: Thüemen 1879.

287. Uromyces viciae-fabae (Pers.) J. Schröt., Hedwigia 14: 98 (1875)

Synonym:- Uromyces fabae (Pers.) de Bary, Grevillea 7(no. 44): 135 (1879)

Ref.: Reichert 1921, Melchers 1931.

288. Uromyces vignae Barclay, J. Asiat. Soc. Bengal, Pt. 2, Nat. Sci. 60(2): 211 (1891) Ref.: Melchers 1931.

289. Uromyces lupini Berk. \& M.A. Curtis, Proc. Amer. Acad. Arts \& Sci. 4: 126 (1860) [1858]

Synonym:- Uromyces lupini Sacc., Nuovo G. bot. ital. 5: 274 (1873)

Ref: Thüemen 1879.

\section{Uropyxidaceae, Pucciniales}

290. Tranz schelia discolor (Fuckel) Tranzschel \& M.A. Litv., J. Bot., Paris 24(3): 248 (1939)

Synonym:- Puccinia pruni-spinosae f. discolor (Fuckel) J.C. Fisch., (1904)

Ref.: Melchers 1931.

291. Tranzschelia pruni-spinosae (Pers.) Dietel, Annls mycol. 20(1/2): 31 (1922)

Synonym:- Puccinia pruni-spinosae f. discolor (Fuckel) J.C. Fisch., (1904)

- Puccinia pruni-spinosae Pers., Syn. meth. fung. (Göttingen) 1: 226 (1801)

Ref. Reichert 1921, Melchers 1931.

\section{Floromycetaceae, Urocystidales}

Basidiomycota, Ustilaginomycetes

292. Antherospora tourneuxii (A.A. Fisch. Waldh.) R. Bauer, M. Lutz, Begerow, Piątek \& Vánky, Mycol. Res. 112(11): 1302 (2008)

Synonym:- Ustilago vaillantii var. tourneuxii A.A. Fisch. Waldh., Verh. bot. Ver. Prov. Brandenb. 22: 65 (1880)

Ref.: Reichert 1921, Melchers 1931.

293. Antherospora vaillantii (Tul. \& C. Tul.) R. Bauer, M. Lutz, Begerow, Piątek \& Vánky, Mycotaxon 110: 293 (2009)

Synonym:- Ustilago vaillantii Tul. \& C. Tul., Annls Sci. Nat., Bot., sér. 3 7: 90 (1847)

Ref.: Thüemen 1880, Roumeguère 1881, Reichert 1921, Melchers 1931.

\section{Urocystidaceae, Urocystidales}

294. Urocystis tritici Körn., Hedwigia 16: 33 (1877)

Ref.: Melchers 1931.

\section{Ustilaginaceae, Ustilaginales}

295. Anthracocystis ehrenbergii (J.G. Kühn) McTaggart \& R.G. Shivas, in McTaggart, Shivas, Geering, Vánky \& Scharaschkin, Persoonia, Mol. Phyl. Evol. Fungi 29: 121 (2012)

Synonym:- Sorosporium ehrenbergii J.G. Kühn, Aperçu Syst. Ustil.: 87 (1877)

Ref.: Melchers 1931.

296. Anthracocystis penniseti (Rabenh.) McTaggart \& R.G. Shivas, in McTaggart,

Shivas, Geering, Vánky \& Scharaschkin, Persoonia, Mol. Phyl. Evol. Fungi 29: 126 (2012)

Synonym:- Sphacelotheca penniseti (Rabenh.) Reichert, Bot. Jb. 56: 85 (1921)

- Ustilago penniseti Rabenh., Hedwigia 10: 18 (1871)

Ref.: Thüemen 1879, Reichert 1921, Melchers 1931.

297. Sporisorium algeriense (Pat.) Vánky, Mycotaxon 65: 174 (1997)

Synonym:- Cintractia algeriensis Pat., Bull. Soc. mycol. Fr. 18: 48 (1902)

Ref.:Reichert 1921. 
298. Sporisorium desertorum (Thüm.) Vánky, Mycotaxon 74(1): 169 (2000)

Synonym:- Sorosporium desertorum Thüm., in Schweinfurth \& Thümen, Grevillea 8 (no. 46): 50 (1879)

Ref.: Thüemen 1879, Reichert 1921, Melchers 1931.

299. Sporisorium egyptiacum (A.A. Fisch. Waldh.) Vánky [as 'aegypticum'], Mycotaxon 33: 371 (1988)

Synonym:- Ustilago egyptiaca A.A. Fisch. Waldh. [as 'aegyptiaca'], Hedwigia 18: 100 (1879)

Ref.: Magnus 1910, Reichert 1921, Melchers 1931.

300. Sporisorium lepturi (Thüm.) Vánky, Mycotaxon 40: 163 (1991)

Synonym:- Ustilago carbo var. lepturi Thüm., in Fischer von Waldheim, Annls Sci. Nat., Bot., sér. 6 4: 200 (1877)

Ref.: Thüemen 1878.

301. Sporisorium sorghi Ehrenb. ex Link, in Willdenow, Sp. pl., Edn 4 6(2): 86 (1825)

Synonym:- Sphacelotheca sorghi (Ehrenb. ex Link) G.P. Clinton, J. Mycol. 8(3): 140 (1902)

Ref.: Thüemen 1879, Reichert 1921, Melchers 1931.

302. Sporisorium tricholaenae (Henn.) Vánky, Mycotaxon 33: 367 (1988)

Synonym:- Ustilago tricholaenae Henn., Bot. Jb. 17: 3 (1893)

Ref.: Reichert 1921, Melchers 1931.

303. Tranzscheliella hypodytes (Schltdl.) Vánky \& McKenzie, Fungal Diversity Res. Ser. 8: 156 (2002)

Synonym:- Ustilago hypodytes (Schltdl.) Fr., Syst. mycol. (Lundae) 3(2): 518 (1832)

Ref.: Thüemen 1880, Reichert 1921, Melchers 1931.

304. Ustilago aschersoniana A.A. Fisch. Waldh., Hedwigia 18(1): 12 (1879)

Ref.: Thüemen 1879, Ascherson 1879, Magnus 1910, Reichert 1921, Melchers 1931.

305. Ustilago avenae (Pers.) Rostr., Overs. K. danske Vidensk. Selsk. Forh. Medlemmers Arbeider: 13 (1890)

Synonym:- Ustilago levis (Kellerm. \& Swingle) Magnus, Verh. bot. Ver. Prov. Brandenb. 37: 96 (1896) [1895]

Ref.:Reichert 1921, Melchers 1931.

306. Ustilago bullata Berk., in Hooker, Bot. Antarct. Voy. Erebus Terror 1839-1843, II, Fl. Nov.-Zeal.: 196 (1855)

Synonym:- Ustilago bromivora (Tul. \& C. Tul.) A.A. Fisch. Waldh., Bull. Soc. Imp. nat. Moscou 40: 252 (1867)

Ref.: Magnus 1910, Reichert 1921, Melchers 1931.

307. Ustilago carbo f. hordei-vulgaris Thüm., Grevillea 6(no. 39): 103 (1878)

Ref: Thüemen 1878.

308. Ustilago carbo Tul. f. tritici- vulgaris Thüemen 1879

Ref. Thüemen (1879).

309. Ustilago cynodontis (Pass.) Henn., Bull. Herb. Boissier 1: 114 (1893)

Ref.: Reichert 1921, Melchers 1931.

310. Ustilago digitariae (Kunze) Rabenhorst, in Klotzsch, Herb. Myc, n. 1199 (1847) et in Flora, XXXIII, pag. 625 (1850).

Ref.: Reichert 1921, Melchers 1931.

311. Ustilago hordei (Pers.) Lagerh., Mitt. bad. bot. Ver.(59): 70 (1889)

Synonym:- Ustilago carbo (DC.) Tul. \& C. Tul., Annls Sci. Nat., Bot., sér. 3 7: 78 (1847)

Ref.: Thüemen 1880, Roumeguère 1881, Reichert 1921, Melchers 1931.

312. Ustilago ischaemi Fuckel, Jb. nassau. Ver. Naturk. 15: 22 (1860)

Ref: Thüemen 1880.

313. Ustilago lepturi Woron., Izv. Kavkaz. Muz. 12: 31 (1919)

Ref.: Reichert 1921, Melchers 1931. 
314. $\quad$ Ustilago lolii Magnus, Hedwigia 49: 93 (1909)

Ref.: Magnus 1910, Reichert 1921, Melchers 1931.

315. Ustilago maydis (DC.) Corda, Icon. fung. (Prague) 5: 3 (1842)

Ref.: Melchers 1931.

316. Ustilago nuda (C.N. Jensen) Rostr., Term. Füz. 8: 745 (1889)

Ref.: Reichert 1921, Melchers 1931.

317. Ustilago residua G.P. Clinton, J. Mycol. 8(3): 133 (1902)

Ref.: Reichert 1921.

318. Ustilago segetum (Bull.) Roussel, Fl. Calvados, Edn 2: 47 (1806)

Ref.: Sickenberger 1901.

319. Ustilago tritici (Pers.) Rostr., Overs. K. danske Vidensk. Selsk. Forh. Medlemmers Arbeider: 15 (1890)

Synonym:- Ustilago schumanniana Henn., Bull. Herb. Boissier 1: 115 (1893)

- Ustilago ehrenbergiana A.A. Fisch. Waldh., Hedwigia 18: 100 (1879)

- Ustilago tritici f. tritici (Pers.) Rostr., Overs. K. danske Vidensk. Selsk. Forh. Medlemmers Arbeider (1890)

Ref.: Reichert 1921, Melchers 1931.

320. Ustilago trichophora (Link) Kunze, Flora, Regensburg 13: 369 (1830)

Ref.: Thüemen 1880, Reichert 1921, Melchers 1931.

Rhizopodaceae, Mucorales

\section{Zygomycota, Incertae sedis}

321. Rhizopus stolonifer (Ehrenb.) Vuill., Revue mycol., Toulouse 24: 54 (1902)

Ref.: Melchers 1931.

\section{Albuginaceae, Albuginales}

Chromista

Oomycota, Peronosporea

322. Albugo candida (Pers.) Roussel, Fl. Calvados, Edn 2: 47 (1806)

Ref.: Thüemen 1878, Magnus 1910, Reichert 1921, Melchers 1931.

323. Pustula tragopogonis (Pers.) Thines, Mycotaxon 92: 455 (2005)

Synonym:- Albugo tragopogonis (Pers.) Gray, Nat. Arr. Brit. Pl. (London) 1: 540 (1821)

Ref.: Reichert 1921.

324. Wilsoniana portulacae (DC.) Thines, Mycotaxon 92: 456 (2005)

Synonym:- Cystopus portulacae (DC.) Lév., Annls Sci. Nat., Bot., sér. 3 8: 371 (1848)

Ref.: Melchers 1931.

\section{Peronosporaceae, Peronosporales}

325. Bremia lactucae Regel, Bot. Ztg. 1: 666 (1843)

Ref.:Reichert 1921, Melchers 1931.

326. Hyaloperonospora parasitica (Pers.) Constant., in Constantinescu \& Fatehi, Nova Hedwigia 74(3-4): 310 (2002)

Synonym:- Peronospora parasitica (Pers.) Fr., Summa veg. Scand., Section Post. (Stockholm): 493 (1849)

Ref.: Melchers 1931.

327. Peronospora alsinearum Casp., Monatsber. Königl. Preuss. Akad. Wiss. Berlin 3: 330 (1855)

Ref.: Thüemen 1880, Reichert 1921, Melchers 1931.

328. Peronospora arborescens (Berk.) de Bary, Annls Sci. Nat., Bot., sér. 4 20: 119 (1863)

Ref.: Melchers 1931.

329. Peronospora destructor (Berk.) Casp. ex Berk., in Berkeley, Outl. Brit.

Fung. (London): 349 (1860)

Ref.: Melchers 1931. 
330. Peronospora farinosa (Fr.) Fr., Summa veg. Scand., Section Post. (Stockholm): 493 (1849)

Ref.: Melchers 1931.

331. Peronospora sparsa Berk., Gard. Chron., London: 308 (1862)

Ref.: Melchers 1931.

332. Peronospora trifoliorum de Bary, Annls Sci. Nat., Bot., sér. 4 20: 117 (1863)

Ref.: Melchers 1931.

333. Peronospora viciae (Berk.) de Bary, Monatsber. Königl. Preuss. Akad. Wiss. Berlin 20: 122 (1863)

Ref.: Melchers 1931.

334. Phytophthora citrophthora (R.E. Sm. \& E.H. Sm.) Leonian, Bot. Gaz. 42: 215, 221 (1906)

Ref.: Melchers 1931.

335. Phytophthora infestans (Mont.) de Bary, J. Roy. Agric. Soc. England, ser. 2 12: 240 (1876)

Ref.: Thüemen 1879, Reichert 1921, Melchers 1931.

336. Plasmopara viticola (Berk. \& M.A. Curtis) Berl. \& De Toni, in Berlese, De Toni \& Fischer, Syll. fung. (Abellini) 7: 239 (1888)

Ref.: Melchers 1931.

337. Pseudoperonospora cubensis (Berk. \& M.A. Curtis) Rostovzev, Annals Inst. Agron. Moscow 9: 47 (1903)

Ref.: Reichert 1921, Melchers 1931.

\section{Pythiaceae, Peronosporales}

338. Globisporangium debaryanum (R. Hesse) Uzuhashi, Tojo \& Kakish., Mycoscience 51(5): 361 (2010)

Synonym:- Pythium debaryanum R. Hesse [as 'de-baryanum'], Pythium debaryanum: ein endophytischer Schmarotzer in den Geweben der Keimlinge der Leindotter, der Rüben, der Spergels und einiger anderer landwirthschaftlichen Kulturpflanzen: 34 (1874)

Ref.: Reichert 1921, Melchers 1931.

339. Sclerospora graminicola (Sacc.) J. Schröt., in Cohn, Krypt.-Fl. Schlesien (Breslau) 3.1(9-16): 236 (1886)

Ref.: Melchers 1931.

\section{Acknowledgements}

The authors would like to thank the anonymous reviewers for their valuable comments and they are also grateful to Dr. Paul Kirk (Royal Botanic Gardens, Kew) for his kind support during the preparation of the manuscript and continous updating of Index Fungorum database by introducing the missed Egyptian taxa.

\section{References}

Abdel-Azeem AM 2010 - The history, fungal biodiversity, conservation, and future perspectives for mycology in Egypt. IMA Fungus 1, 123-142.

Abdel-Azeem AM, Salem Fatma M. 2013 - A checklist of Egyptian fungi: I. Protozoan fungal analogues. Mycosphere 4(4), 794-807.

Ascherson P. 1879 - Beitrag zur Flora Aegyptens. Berlin.

Bishara I. 1928 - Cotton insects. Ministry of Agriculture, Egypt, Monthly Report 1: 4.

Briton-Jones HR. 1922- The smuts of millet. Ministry of Agriculture, Egypt. Technical Series Bulletin 18, 1-9.

Briton-Jones HR. 1923 - A wound parasite of cotton bolls. Ministry of Agriculture, Egypt.Technical Series Bulletin 19, 1-8. 
Briton-Jones HR. 1925 - Mycological work in Egypt during the period 1920-1922. Ministry of Agriculture, Egypt. Technical Series Bulletin 49, 1-129.

Decaisne, MJ. 1835 - Notice sur quelques plantes de la Flore d'Egypte. Annales des Sciences Naturelles Botanique 4, 193-208.

Delile AR. 1813 - Flore d'Egypte. Paris.

El-Abyad MS, Abu-Taleb A. 1993 - II-Soil Fungi. Ecology of fungal flora. In: Publication of National Biodiversity Unit. No. 1. Habitat Diversity: 237-262. Egyptian Environmental Affairs Agency, Egypt.

El-Abyad MS. 1997 - Biodiversity of Fungal Biota in Egypt. Up-dated check-list. [Publication of National Biodiversity Unit No. 7.] Egyptian Environmental Affairs Agency.

Fahmy T. 1923 - The production by Fusarium solani of a toxic excretory substance capable of causing wilting in plants. Phytopathology 13, 543.

Fletcher F. 1902 - Notes on two diseases of cotton. Journal of Khedive Agricultural Society and School of Agriculture Giza, Egypt 4, 2.

Hyde KD, Jones EBG, Lui J-K, Ariyawansa H, Boehm E, et al. 2013 - Families of Dothideomycetes. Fungal Diversity 63, 1-313.

Kirk PM, Ansell AE. 1992 - Authors of Fungal Names. Kew: CAB International, 95 p.

Kirk PM, Cannon PF, Minter DW, Stalpers JA. 2008 - Ainsworth \& Bisby's dictionary of the fungi. $10^{\text {th }}$ edn, Wallingford: CAB International.

Magnus P. 1910 - Beitrag zur Kenntnis der parasitischen Pilze Ägyptens. Hedwigia XLIX, S. 93

Melchers LE. 1931 - A check list of plant diseases and fungi occurring in Egypt. Transactions of the Kansas Academy of Science 34, 41-106.

Moustafa AF, Abdel-Azeem AM. 2011 - An annotated check-list of Ascomycota reported from soil and other terricolous substrates in Egypt. Journal of Basic and Applied Mycology 2, 127.

Patouillard NT. 1895 - Quelques espèces nouvelles de Champignons africains. Bulletin de la Société Mycologique de France 11, 85-88.

Reichert I. 1921 - DiePilzflora Äegypten. Engler's Botanischen Jahrbüchen 56, 595-727.

Roumeguère C. 1881 - Champignon rapportés en 1880 d'une excursion botabique en Egypte et en Palestine par M. William Barbay. Revue Mycologique Toulouse 3, 23-25

Roumeguère C. 1887 - Champignons d'Egypte. Revue Mycologique 36: 205-207.

Shearer A. 1924 - Cotton wilt $3^{\text {rd }}$ Annual Report, Cotton Research Board, Ministry of Agriculture. Egypt: 37.

Sickenberger E. 1901 - Contribution à la Flore d'Egypte. Mémoires de l'institut Egypte 4901, p. 331-332.

Söderström L, Hagborg A, von Konrat M, Renner MAM. 2008 - Early Land Plants Today: Liverwort checklist of checklists. Fieldiana, Botany, n.s. 47, 105-130.

Söderström L, Urmi E, Ván̆a J. 2007 - The distribution of Hepaticae and Anthocerotae in Europe and Macaronesia- Update 1-427. Cryptogamie, Bryologie 28, 299-350.

Thümen F. 1878 - Fungi Egyptiaci, Ser. I. Grevillea 6 (39), 102-104.

Thümen F. 1879 - Fungi Egyptiaci, Ser. II. Grevillea 8 (46), 49-51.

Thümen F. 1880 - Fungi Egyptiaci, Ser. III. Flora 63 (30), 477-479.

Wijayawardene NN, Crous PW, Kirk PM, Hawksworth DL, Boonmee S, Braun U, et al. 2014 Naming and outline of Dothideomycetes including proposals for the protection or suppression of generic names. Fungal Divers. 2014, 1-55.

www.indexfungorum.org- December 2015. 